\title{
Experimental study on the recovery of MVC and muscle power after muscle fatigue with different recovery methods
}

\author{
Fan Zhang ${ }^{1,2, \text { a }}$ \\ ${ }^{1}$ Department of Police Skills and Tactics, Nanjing Forest Police College, Nanjing 210023, China; \\ ${ }^{2}$ Sports Science postdoctoral programme, Nanjing Normal University, Nanjing 210023, China. \\ azhangfan@nfpc.edu.cn
}

Keywords: MVC; musle power; muscle fatigue recovery; recovery effect.

\begin{abstract}
This paper's purpose is to investigate the effect of passive recovery (PR), active recovery (AR), vibration recovery (VR) and cryotherapy recovery (CR) on muscle performance(Maximum Voluntary Contraction, muscle power) after muscle fatigue. 20 healthy male college students were enrolled in the study. MVC and MP of the biceps brachii of the non-handedness were measured. The differences and recovery effects of MVC and MP at different detection time points were tested by one-way repeated measures ANOVA. Results shows that $10 \mathrm{~min}$ VR and CR had about 15\% muscle power recovery effect. In view of this, it can be inferred that both CR and VR can effectively help the recovery of muscle strength after muscle fatigue, but there is no difference between the 2 groups.
\end{abstract}

\section{Introduction}

Exercise is quite beneficial to physical and psychological health, but overexercise or game with furious competition may cause physical fatigue, and especially, muscle fatigue sourced from continuous muscle contraction will lower late exercise performance and bring muscle injury response [1], and therefore, if effective recovery method is used within limited time, it will be quite beneficial to re-performance improvement and exercise injury reduction.Given above content, this research takes biceps brachii muscle (BBM) that is fatigable in exercise as object to discuss effect of different recovery methods, such as passive recovery (PR), active recovery (AR), vibration recovery (VR) and cryotherapy recovery (CR) etc., on muscle performance recovery effect and difference under muscle fatigue condition, so as to provide scientific reference for exercise performance re-improvement and injury reduction under muscle fatigue condition.

\section{Research object and method}

\subsection{Object of study}

20 general male undergraduates having healthy body are subjects (they are free from cardiovascular disease, high blood pressure and relevant viscera disease, and they can receive the maximum exercise test), with average age (20.4 \pm 2.3 yrs.), average height $(173.5 \pm 3.4 \mathrm{~cm})$ and average weight $(68.7 \pm 3.9 \mathrm{~kg})$.

\subsection{Experimental design.}

Experimental design of repetitive measurement is adopted, and every subject accepts four different fatigue recovery ways in anti-balance sequence method: PR, AR, VR, CR. Interval of experimental procedure in every step shall be over 7 days to avoid that delayed onset muscle soreness disturbs experimental result.

1) In phase of familiarization with actions, elbow joint shall bend to contract 10 times centripetally and eccentrically repeatedly in $45 \%$ s of angular speed;

2) In phase of measurement before fatigue, BBM maximum voluntary contraction (MVC), muscle power (MP), shall be measured in sequence to avoid that mechanical property is affected by muscle manifestation test. 
3) In phase of muscle fatigue, isokinetic exercise system of Biodex System Pro-4 is adopted, and maximum contraction exercise shall be conducted centripetally and eccentrically repeatedly in $45^{\circ} / \mathrm{s}$ of angular speed on bent of non-habitual elbow joint, and activity scope is $30 \sim 120^{\circ}$ (it is $0^{\circ}$ when elbow completely stretches straightly), and muscles can be judged in fatigue until finally there are continual three times where muscle force manifestation reduces to $50 \% \mathrm{MVC}$, and then exercise of constant speed can be stopped.

4) In phase of measurement after fatigue, data of MVC and MP shall be immediately measured.

5) In phase of intervening of recovery way, one of its recovery ways can be conducted in random way: PR group shall rest for 10 minutes in still sitting gesture; concentric contraction exercise shall be conducted for AR group in constant speed on isokinetic exercise system of Biodex System Pro-4. It shall start in strength of 25\% MVC, and the speed shall decrease by 5\% MVC every two minutes, and there shall be 10 minutes totally. For VR group, vibration training platform Power Plate Pro5 AIR ${ }^{\mathrm{TM}}$ shall be used to partially vibrate BBM, and vibration frequency shall be $30 \mathrm{~Hz}$, amplitude being $2 \mathrm{~mm}$ for 10 minutes. Ice compression shall be conducted on fatigue parts with LP ice pack (LP894; M: $22.9 \mathrm{~cm}$; 65\% ice block filling) non-pressure device for 10 minutes for CR group.

6) In phase of measurement after recovery, MVC and MP of BBM shall be measured immediately.

7) Then experiment of another recovery way shall be conducted until totally recovery with interval over 7 days.

\subsection{Test data and collection.}

1) Maximum Voluntary Contraction and muscle power. MVC and MP of elbow joint can be measured via Biodex System Pro-4. Then maximum isometric voluntary contraction can be conducted for three times with elbow joint bending at $70^{\circ}$, and duration of every contraction shall be 3 sounds with interval at 30 sounds, and then mean value during 2 sounds of each contraction can be chosen as MVC (Nm). Mean value of isometric contraction (strength change after 250 ms of every contraction) for three times can be chosen as MP $(\mathrm{Nm} / \mathrm{s})$. Collected signal via isokinetic exercise system shall be transferred to recorder (BIOPACK systems MP150) and then delivered to software of Acqknowledge 4.3 for analysis (smoothing shall be conducted via low-pass $50 \mathrm{~Hz}$ after amplification of physiological amplifier).

2) Recovery effect. Values of all parameters (MVC, MP) at different measurement time points (before fatigue, after fatigue and after recovery) shall be calculated, and then [(after recovery/before fatigue) $\times 100 \%$-(after fatigue/before fatigue) $\times 100 \%$ ] shall be deemed as recovery effect of all parameters.

\subsection{Statistical analysis.}

Statistical handling shall be conducted via SPSS 19.0, and the result shall be represented with mean value \pm standard deviation ( $\bar{X} \pm \mathrm{SD}$ ). Difference of MVC and MP in all groups (PR, AR, VR, CR) at different time points (before fatigue, after fatigue, after recovery) shall be compared respectively via repeated measures of a single factor. Difference among groups of recovery effect can also be compared, and posterior comparison can be conducted via way of Bonferroni if standard reaching is significant. Statistical significance level is $P<0.05$, and non-significance level is $P<0.01$.

\section{Change of MVC and MP after intervening of different recovery ways}

\subsection{Difference of different time points.}

Significance difference $(P<0.05)$ of MVC and MP in all groups at different time points is reached; after posterior comparison (Table 1), MVC of all groups after fatigue is significantly less than that before fatigue $(P<0.05)$. There is no significant difference $(P>0.05)$ among groups after fatigue, while MVC of VR and CR groups after fatigue are both significantly more than that after fatigue $(P<0.05)$. 
Table 1. MVC and MP at different time points

\begin{tabular}{cccc}
\hline $\begin{array}{c}\text { MVC(Nm)/ } \\
\text { MP(Nm/s) }\end{array}$ & before fatigue & after fatigue & after recovery \\
\hline PR & $40.13 \pm 7.76$ & $27.89 \pm 9.12^{\mathrm{a}} / 91.90 \pm 23.22^{\mathrm{a}}$ & $30.04 \pm 8.28^{\mathrm{b}} / 119.06 \pm 21.31^{\mathrm{bc}}$ \\
AR & $39.46 \pm 7.57$ & $27.13 \pm 9.60^{\mathrm{a}} / 85.47 \pm 25.13^{\mathrm{a}}$ & $25.47 \pm 14.76^{\mathrm{b}} / 98.94 \pm 42.76^{\mathrm{b}}$ \\
VR & $39.04 \pm 7.23$ & $27.82 \pm 8.66^{\mathrm{a}} / 85.61 \pm 22.70^{\mathrm{a}}$ & $33.27 \pm 9.50^{\mathrm{bc}} / 122.67 \pm 27.29^{\mathrm{bc}}$ \\
CR & $38.61 \pm 9.05$ & $25.96 \pm 8.79^{\mathrm{a}} / 87.77 \pm 33.19^{\mathrm{a}}$ & $32.49 \pm 8.78^{\mathrm{bc}} / 127.07 \pm 25.99^{\mathrm{bc}}$ \\
\hline
\end{tabular}

Note:a represents significant difference before fatigue and after fatigue; b represents significant difference before fatigue and after recovery; c represents significant difference after fatigue and recovery. $\mathrm{P}<0.05$.

\subsection{Difference of recovery effect.}

MVC and MP recovery effect among all groups reaches significant difference $(P<0.05)$, and CR is significantly superior to PR and AR $(P<0.05)$ after posterior comparisons (Fig. 1$)$. VR is significantly more than AR $(P<0.05)$ after posterior comparison (Fig. 2$)$.

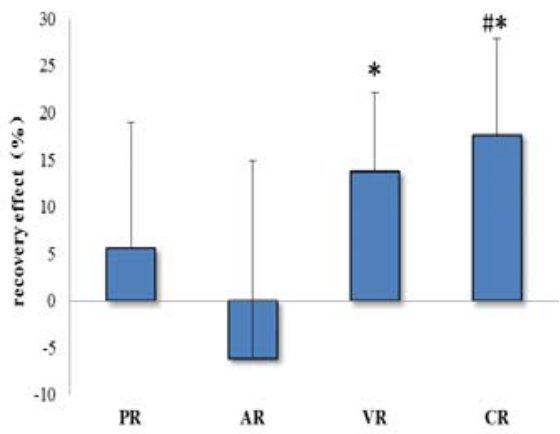

Fig.1 Comparison of the recovery effect of MVC Fig.2 Comparison of the recovery effect of MP Note: \# represents significantly better than group PR; * represents was higher than that of AR group $(\mathrm{p}<0.05)$; \& represents was better than that of group $\mathrm{CR}$. $\mathrm{P}<0.05$

\section{Summary}

It is proved that exercise performances such as MVC and MP can be effectively enhanced via vibration stimulus according to experimental result. Though subjects mostly accepted vibration stimulus on condition of non-fatigue in past researches, Jordan, etc [2] pointed out that there is good effect on power output performance if vibration stimulus is imposed on fatigue state of muscles, indicating that vibration stimulus can not only enhance power performance of non-fatiguing muscles, but also it can strengthen performance of MP and MVC for muscles in state of fatigue. Vibration stimulus can improve mechanism of power performance, and the reason may be that vibration stimulus can promote activation of nervous centralis and increase motor neural excitation and nervous impulse, synchronization of motor unit, and enhance degrees of myotatic reflex and muscle activation and restriction on rivalry muscles, etc, so it can effectively improve exercise performance.

It is indicated in experimental result that CR can significantly improve power recovery after fatigue. It was also found in past researches that immediate CR can effectively recover strength performance, for example, muscle force recovery could be boosted via 15 min CR after tiring exercise. Even strength could be recovered and exercise performance could be boosted meanwhile via 3 minutes of CR arms and shoulders. Its function mechanism may be as what scholars such as Sramek, etc explained: CR improving exercise performance may be related to enhancing activation of sympathicus to maintain body temperature in low temperature and increasing adrenaline as well as heart rate. If muscle temperature is relatively high, then it is likely to cause consumption of over much energy (such as sugar and phosphate, etc). So it is also indicated in this research that it can not only reduce muscle temperature, but also it can reduce energy consumption at the time of fatigue recovery and greatly benefit improving following exercise performance to give CR immediately after muscle 
fatigue. However, what needs to be pointed out is that it is found in researches[3] that muscle fatigue on the third day after CR is significantly higher than that without CR. It indicates that CR can effectively reduce muscle injury and inflammatory reaction after centrifugal motion, bit it can also increase muscle injury and delay muscle fatigue. It was said in a research[4] that partial CR intervening has no effect on recovery of BBM injury in February of 2017. Obviously, it shall be cautious to design CR time and temperature, besides, it is recommended in related researches[5] that CR time had better be not over 20 minutes whether adding pressure or not.

It is found in this research that AR is not very effective on recovery of strength, though it has been proved in many researches that AR can boost blood circulation and promote anaerobic metabolism, it has different effect on performance of re-exercise. It is indicated in past researches that oxygen demand of AR is higher than PR, so oxygen composition of myoglobin and hemoglobin is reduced. Human body will continuously consume energy and reduce storage of ATP-PCr, causing bad effect on performance of re-exercise. Though Dupont, etc[6] points out that short AR (15 120 s) has bad effect on recovery of exercise performance, AR recovery effect can be improved as the duration increases. But Mcainch, etc find that AR (40\% of maximum oxygen intake peak) has bad effect on exercise performance after exhausted exercise, and ATP-PCr for AR is relatively less than that for PR. Besides, it is pointed out by Linnane, etc that high muscle temperature can accelerate glycogen decomposition rate, causing quick fatigue and output reduction of average strength. However, AR is only conducted on elbow joint for 10 minutes in this research, so it mostly belongs to aerobic exercise for general or big muscle group. It can only exert limited function on cycle metabolism and storage as well as recovery of oxygen and ATP-PCr, so it needs to be further researched in future work.

\section{Acknowledgments}

This work was supported in part by the Project of the Fundamental Research Funds for the Central Universities under Grant LGZD201709, in part by the Project of China Postdoctoral Science Foundation under Grant 2017M611849, in part by Jiangsu Qing LAN Project under Grant 2017, and in part by Nanjing Forest police College Teaching Reform Project under Grant ZD17001 \& YB17001.

\section{References}

[1]. Ibrahim O, Omar H, Hamdi C, et al. Effects of Recovery Type after a Kickboxing Match on Blood Lactate and Performance in Anaerobic Tests. Asian Journal of Sports Medicine. Vol. 5 (2014) No. 2, p. 99-107.

[2]. Jordan M J, Norris S R, Smith D J, et al. Vibration training: an overview of the area, training consequences, and future considerations. Journal of Strength \& Conditioning Research. Vol. 19 (2015) No. 2, p. 459-466.

[3]. Burgess T L, Lambert M I. The efficacy of cryotherapy on recovery following exercise-induced muscle damage. International Sport med Journal. Vol. 11 (2010) No. 2, p. 258-277.

[4]. Lima C S, Medeiros D M, Prado L R, et al. Local cryotherapy is ineffective in accelerating recovery from exercise-induced muscle damage on biceps brachii. Sport Sciences for Health. Vol. 2 (2017) No. 22, p. 1-7.

[5]. Santos V B D C, Cardoso C D S, Figueiredo C P, et al. Effect of cryotherapy on the ankle temperature in athletes: ice pack and cold water immersion. Fisioter Mov. Vol. 28 (2015) No. 1, p. 23-30.

[6]. Dupont G, Blondel N, Berthoin S. Performance for short intermittent runs: active recovery vs. passive recovery. European Journal of Applied Physiology. Vol. 89 (2003) No. 6, p.548-554. 